\title{
Rückforderung von BerufungsLeistungsbezügen bei vorzeitigem Hochschulwechsel - BayVGH, Urt. vom 18.08.2017, 3 BV 16.132
}

$\begin{aligned} \text { Authors: } & \text { Frank Wertheimer } \\ \text { Submitted: } & \text { 26. March } 2019 \\ \text { Published: } & 25 . \text { April } 2019 \\ \text { Volume: } & 6 \\ \text { Issue: } & 4 \\ \text { Affiliation: } & \text { Krauss Law, Lahr, Germany } \\ \text { Languages: } & \text { German } \\ \text { Keywords: } & \text { Law, social, college, university } \\ \text { Categories: } & \text { Humanities, Social Sciences and Law } \\ \text { DOI: } & 10.17160 / \text { josha.6.4.549 }\end{aligned}$

Abstract:

$\mathrm{n}$ the proceedings, the Bayerische Verwaltungsgerichtshof (Bavarian Administrative Court) had to deal with the question whether a university may reclaim from a professor benefit which had been promised and granted to her in connection with her appointment to a fixed-term professorship if the professor changes to another university within three years. This repayment obligation was referred to in the university's appeal offer. The Administrative Court considered the reclaiming of the remuneration for the appeal benefits to be unlawful but took the view that a reclaiming of the remuneration for the appeal benefits could be permissible in the case of indefinite remuneration for the stay. Previously published in Ordnung der Wissenschaft 2018, ISBN/ISSN 3-45678-222-7

\section{JOSHA Jamana osemene, Humanities and Arts}




\title{
Rückforderung von Berufungs-
}

Leistungsbezügen bei vorzeitigem

Hochschulwechsel - BayVGH, Urt. vom

18.08.2017, 3 BV 16.132

\section{Frank Wertheimer}

\begin{abstract}
$\underline{\text { Abstract }}$
In dem Verfahren hatte sich der Bayerische Verwaltungsgerichtshof mit der Frage auseinanderzusetzen, ob eine Hochschule von einer Professorin Leistungsbezüge, die ihr im Rahmen ihrer Berufung auf eine befristete Professur befristet zugesagt und gewährt wurden, zurückverlangen kann, wenn die Professorin innerhalb von drei Jahren an eine andere Hochschule wechselt. Auf diese Rückzahlungsverpflichtung war im Berufungsangebot der Hochschule hingewiesen worden. Der Verwaltungsgerichtshof erachtete die Rückforderung der Berufungsleistungsbezüge für rechtswidrig, vertrat dabei aber die Auffassung, dass eine Rückforderung im Falle von unbefristeten Bleibe-Leistungsbezügen zulässig sein könne.
\end{abstract}

Previously published in Ordnung der Wissenschaft 2018, ISBN/ISSN 3-45678-222-7 


\section{Die Fallkonstellation}

Die Klägerin war mit Wirkung vom 01.04.2012 unter Berufung in ein Beamtenverhältnis auf Zeit für die Dauer von 5 Jahren zur Universitätsprofessorin der Besoldungsgruppe W2 ernannt worden. Das Berufungsangebot der Hochschule enthielt die Zusage eines Berufungsleistungsbezuges in Höhe von 1.500,00 EUR, der aufschiebend bedingt unbefristet gewährt wurde, ferner einen weiteren - befristeten - Berufungsleistungsbezug in Höhe von 600,00 EUR. Das von der Klägerin angenommene Berufungsangebot enthielt am Ende den Hinweis, dass die gewährten Leistungsbezüge nach Maßgabe der Vergabegrundsätze der Universität in voller Höhe zurückzuzahlen sind, wenn innerhalb von drei Jahren seit Gewährung ein Wechsel an eine andere Hochschule erfolgt.

Mit Wirkung vom 01.01.2014 wurde die Klägerin unter Berufung in ein Beamtenverhältnis auf Lebenszeit auf eine W3 Professur an einer anderen bayerischen Hochschule ernannt. Mit Bescheid vom 14.01.2014 stellte die frühere Hochschule gegenüber der Klägerin fest, dass sie - weil die 3-Jahresfrist noch nicht abgelaufen sei - zur Rückzahlung der seit Dienstantritt gewährten Leistungsbezüge in voller Höhe verpflichtet sei, ferner, dass die Berufungsleistungsbezüge vom Landesamt für Finanzen zurückgefordert würden. Die Klägerin erhielt daraufhin im April 2014 von dort eine entsprechende Zahlungsaufforderung über 42.290,35 EUR.

Hinsichtlich der Rückzahlung der Leistungsbezüge berief sich die Hochschule auf Art. 70 Abs. 3 S. 2 BayBesG iVm § 3 Abs. 4 S. 2 der Bayerischen Hochschulleistungsbezüge-Verordnung (BayHLeistBV). Danach kann die Hochschule festlegen, dass Berufungs- und Bleibeleistungsbezüge zurückzuzahlen sind, wenn der Professor oder die Professorin innerhalb von drei Jahren seit Gewährung dieser Leistungsbezüge an eine andere Hochschule wechselt.

\section{Entscheidung der Vorinstanz}

Nach erfolglosem Widerspruchsverfahren hatte die Hochschullehrerin Klage gegen den Rückforderungsbescheid erhoben, die das VG Würzburg (W $1 \mathrm{~K}$ 14.811) mit Urteil vom 24.11.2015 abgewiesen hat. Das Verwaltungsgericht sah die Verpflichtung der Klägerin zur Rückforderung der Leistungsbezüge durch die Regelungen des Bayerischen Besoldungsrechts gedeckt. Es wies dabei darauf hin, dass ihm bezüglich der hier streitigen Konstellation keine Gerichtsentscheidungen bekannt seien, die sich mit dieser Thematik in der Vergangenheit 
befasst hätten. In Anknüpfung an die Rechtsprechung zur Rückforderung von Anwärterbezügen bzw. Anwärtersonderzuschlägen bei Nichteinhaltung einer bestimmten Mindestdienstzeit nach Beendigung der Ausbildung ${ }^{1}$ hielt das Verwaltungsgericht die Rückforderung für rechtmäßig. Verstöße gegen Art. 33 Abs. 2 GG, 12 Abs. 1 GG oder Art. 5 Abs. 3 GG verneinte das Gericht. Der Feststellungsbescheid der Hochschule scheitere auch nicht am Grundsatz der Verhältnismäßigkeit in Bezug auf die volle Rückzahlungsverpflichtung, da das Landesamt für Finanzen im anschließenden Verwaltungsverfahren der eigentlichen Rückforderung einer verfassungskonformen Rechtsanwendung Rechnung tragen könne; hier könnten Billigkeitserwägungen nach $\S 15$ Abs. 2 S. 3 BayBesG angestellt werden, auch könne dort dem Gesichtspunkt Bedeutung zukommen, dass eine verfassungsgemäße Alimentation der Klägerin - ohne die Leistungsbezüge - „mit hoher Wahrscheinlichkeit“ durch das gewährte W2-Grundgehalt nicht gewährleistet gewesen sei.

\section{Praktische Bedeutung der Entscheidung des BayVGH}

Mit dem Berufungsurteil vom 18.08.2017 hat der Bayerische Verwaltungsgerichtshof Neuland betreten, weil bislang weder obergerichtliche noch höchstrichterliche Rechtsprechung zu der Frage vorlag, ob und in welchem Umfang eine Hochschule in einer derartigen Konstellation Berufungsleistungsbezüge zurückfordern kann. Das kommt in der Entscheidung auch insoweit zum Ausdruck, als das Gericht die Revision zugelassen hat, weil die Rechtssache grundsätzliche Bedeutung habe. Der Freistaat Bayern legte keine Revision ein, die Entscheidung ist rechtskräftig.

Der Interessenwiderstreit ist evident: Die Hochschulen führen ihren Aufwand, den sie mit einem universitären Berufungsverfahren betreiben sowie die Investitionen, die mit einer Berufungszusage verbunden sind, ins Feld, mit dem Rückforderungsvorbehalt könne ein gewisse Verlässlichkeit gewährleistet werden, dass der bzw. die Berufene eine kalkulierbare Zeit an der Hochschule verbleibe. Aus Sicht der Professorin bzw. des Professors stellt sich die Situation anders dar: In der Verhandlungsphase über die Rufannahme besteht praktisch keine Chance durchzusetzen, dass die Hochschule auf einen Rückforderungsvorbehalt verzichtet, zumal die Ruferteilung noch keine gesicherte Rechtsposition verschafft ${ }^{2}$. Ist die Berufung mit einem Rückforderungsvorbehalt belastet, wird ein Wechsel an eine andere Hochschule erschwert. Vor allem in Konstellationen, in denen - wie hier - ein Wechsel von einer befristeten W2-Professur ohne konkrete Entfristungsoption auf eine höher dotierte

\footnotetext{
$1 \quad$ Unter Verweis auf BVerfG v. 03.07.2007, 2 BvR 733/06, juris; BVerwG v. 27.02.1992, 2 C 28/91, , NVwZ 1993, 372 und BVerwG v. 13.09.2001, 2 A 9/00, ZTR 2002, 95.

2 Vgl. hierzu Wertheimer, OdW 2015, 147 mwN; Detmer in: HSchR-Praxishandbuch, 3. Aufl. 2017, Kap. 4 Rn. $101 \mathrm{f}$.
} 
W3-Lebenszeitprofessur möglich ist, kann sich eine drohende Rückzahlungsverpflichtung nachteilig auswirken.

\section{Urteil des BayVGH vom 18.08.2017}

Der Bayerische Verwaltungsgerichtshof hielt den Bescheid der Hochschule über die Feststellung der Rückzahlung von Berufungs-Leistungsbezügen für rechtswidrig, dieser verletze die Professorin in ihren Rechten ( $\$ 113$ Abs. 1 S. 1 VwGO). In dem Urteil finden sich folgende Aussagen:

1. Ermächtigungsgrundlage für die Rückforderung von Berufungsleistungsbezügen könne allein Art. 70 Abs. 3 S. 2 BayBesG sein. Diese Norm berechtige grundsätzlich zum Erlass eines belastenden Verwaltungsaktes, mit dem gewährte Leistungsbezüge unter den dort genannten Voraussetzungen zurückverlangt werden könnten. In diesem Zusammenhang stellt das Gericht klar, dass Art. 70 Abs. 3 S. 2 zu einer Einzelfallregelung ermächtige, nicht hingegen zu einem entsprechenden Normerlass, so dass dem auf Art. 74 BayBesG beruhenden gleichlautenden $\S 3$ Abs. 4 S. 2 BayHLeistBV keine eigenständige Bedeutung zukommt.

2. Der am Ende des Berufungsangebots enthaltene Hinweis auf die Rückzahlungsverpflichtung unterliege nicht der Inhaltskontrolle gem. § 307 BGB, weil es sich bei der Zusage der Berufungsleistungsbezüge um eine Zusage analog § 38 BayVwVfG handele und nicht um ein Angebot auf Abschluss eines öffentlich-rechtlichen Vertrages.

3. Art. 70 Abs. 3 S. 2 BayBesG gelte nur für unbefristete Berufungs- und BleibeLeistungsbezüge, ein Wille des Gesetzgebers, auch befristet bewilligte Leistungsbezüge mit der Rückforderungsbestimmung zu erfassen, komme nicht rechtssicher zum Ausdruck. Der Verwaltungsgerichtshof schließt dies sowohl aus einer systematischen Binnenauslegung der Vorschrift wie auch aus einer systematischen Vergleichsbetrachtung mit Art. 71 Abs. 2 S. 3 BayBesG, der ebenfalls nur bei unbefristeten Leistungsbezügen für den Fall eines erheblichen Leistungsabfalls des Hochschullehrers eine Widerrufsmöglichkeit vorsieht.

4. Art. 70 Abs. 3 S. 2 BayBesG auf alle Fälle anzuwenden, in denen ein Professor oder eine Professorin innerhalb von drei Jahren seit Gewährung der Berufungs-Leistungsbezüge an eine andere Hochschule wechselt, sei mit höherrangigem Recht nicht vereinbar, weshalb die Vorschrift einer verfassungskonformen Auslegung bedürfe. Werden Berufungsleistungsbezüge für eine nach Ernennung im Dienstverhältnis erbrachte Leistung gezahlt, bestehe für eine Rückzahlungspflicht bei einem Verlassen der Hochschule kein Raum. 
Gefolgert wird dies aus Art. 12 GG. Entgegen der Auffassung des VG Würzburg in der Vorinstanz könne die Rechtsprechung zur Rückzahlung von Ausbildungsaufwendungen ${ }^{3}$ nicht übertragen werden. Mit der Gewährung von Leistungsbezügen habe die Hochschule nicht in die Klägerin investiert, sondern ihr auf Basis ihrer Vergabegrundsätze eine erhöhte Vergütung dafür zugestanden, weil sie schon mit Dienstantritt in der Lage gewesen sei, Leistungen zu erbringen, die die internationale Reputation der Universität entscheidend mitprägten ${ }^{4}$. In einer solchen Situation bestehe kein Vorrang der Belange der Universität vor dem Interesse des befristet beschäftigten Hochschullehrers, Karrierechancen auf eine unbefristete Professur wahrnehmen zu können. Die Belange der Universität, Planungssicherheit $z u$ erhalten und den Aufwand von Berufungsverfahren in einem angemessenen Rahmen zu halten, stellten zwar dem Grunde nach sachgerechte und vernünftige Erwägungen des Gemeinwohls dar, diese lassen jedoch die Einschränkung der Berufsfreiheit von befristet beschäftigten Hochschullehrern nicht als verhältnismäßig erscheinen. Vielmehr seien die arbeitsrechtlichen Grundlagen heranzuziehen, nach denen für Sonderzahlungen mit Entgeltcharakter Rückzahlungsvorbehalte unzulässig sind, wenn Gegenstand der Zahlung Entgelt für bereits geleistete Arbeit ist.

5. Der Senat hält rechtlich unbedenkliche Anwendungsfälle der Ermächtigungsgrundlage des Art. 70 Abs. 3 S. 2 BayBesG für möglich. So könne etwa die Rückforderung von unbefristeten Bleibe-Leistungsbezügen in Betracht kommen, weil diese nicht ohne weiteres als Gegenleistung für geleistete Dienste anzusehen sein werden, sondern auch allein deshalb erbracht werden können, um den (möglicherweise) abwanderungswilligen Hochschullehrer davon abzuhalten, dem Ruf einer anderen Universität zu folgen.

6. Der Umstand, dass die Klägerin bereits einen Anteil des Dreijahreszeitraums tatsächlich abgeleistet habe, könne entgegen der Auffassung der Vorinstanz nicht in dem sich anschließenden Verwaltungsverfahren durch eine Teilrückzahlung nach $\S 15$ Abs. 2 BayBesG berücksichtigt werden, weil es sich bei dieser Vorschrift lediglich um eine Rechtsfolgenverweisung handele. Offen ließ das Gericht in diesem Zusammenhang, ob in einer solchen Fallkonstellation auch eine zeitanteilige Abschmelzung des Rückforderungsbetrages in Betracht komme. 


\section{Bewertung der Entscheidung}

Die dem Fall zugrunde liegende Frage, ob die beklagte Hochschule die befristet gewährten Berufungs-Leistungsbezügen auf Basis des Art. 70 Abs. 3 S. 2 BayBesG zurückverlangen konnte, hat der Bayerische Verwaltungsgerichtshof richtig entschieden. Eine nähere Befassung mit den Urteilsgründen lohnt insbesondere deshalb, weil die Fallkonstellationen bei der Gewährung von Berufungs- oder Bleibe-Leistungsbezügen auch anders gelagert sein können und Antworten auf die dann vorzunehmende rechtliche Bewertung verlangen.

\section{Art. 70 Abs. 3 S. 2 BayBesG als Ermächtigungsgrundlage}

Dass der Verwaltungsgerichtshof Art. 70 Abs. 3 S. 2 BayBesG als alleinige Ermächtigungsgrundlage für die Rückforderung der Leistungsbezüge betrachtet und diese Norm als Ermächtigung zu einer Einzelfallregelung wertet, ist auf Basis der Gesetzgebungsmaterialien ${ }^{5}$ überzeugend begründet. Dem wortgleichen $\S 3$ Abs. 4 S. 2 BayHLeistBV kommt damit keine eigenständige Bedeutung zu.

Die Gesetzesbegründung, wonach Art. 70 Abs. 3 S. 2 BayBesG dem Schutz der Hochschule „vor einem unlauteren Abwerbeverhalten anderer Hochschulen“ diene, ist dabei kritisch zu sehen. Bietet, wie im vorliegenden Fall, eine andere Hochschule einer befristet berufenen Professorin eine höher besoldete Lebenszeitprofessur an, so kann darin kein unlauteres Abwerbeverhalten gesehen werden. Nicht einmal die verfassungsmäßig kritisch zu beurteilende Vereinbarung der Kultusministerkonferenz zur Besetzung von C4- und W3Professuren $^{6}$ hätte hier zu einem Absehen von einer Berufung ${ }^{7}$ geführt. Abgesehen davon begegnet die Argumentation der bayerischen Legislative auch systematischen Bedenken: Es passt nicht zusammen, dass mit der Vorschrift ein unlauteres Abwerbeverhalten einer anderen Hochschule verhindert werden soll, die "Zeche" aber der betreffende Hochschullehrer bezahlen soll, der die Hochschule innerhalb des Dreijahreszeitraums verlässt. Andere Bundesländer verzichten daher auch auf ein derartiges nicht nur rechtlich, sondern auch hochschulpolitisch fragwürdiges Instrument.

In diesem Zusammenhang enthalten die Urteilsgründe noch eine kleine Ranke. Der Hinweis im Berufungsschreiben auf die Rückzahlungsverpflichtung unterliege, so der Verwaltungsgerichtshof, nicht einer Inhaltskontrolle nach $\S 307$ BGB, weil es sich bei der Zusage der Hochschulleistungsbezüge um eine Zusage analog $\S 38$ BayVwVfG handelt und

LT-Drs. 16/3200 S. 418.

KMK-Vereinbarung über die Besetzung von Professorinnen und Professoren an den Hochschulen, Beschluss vom 10.11.1978 i.d.F. vom 15.08.2002; siehe hierzu Detmer, a.a.O., Kap. IV, Rn. 77.

Abschnitt II Nr. 3 Abs. 2 der KMK-Vereinbarung. 
nicht um ein Angebot auf Abschluss eines öffentlich-rechtlichen Vertrages. Stellt man auf die äußeren Umstände $a b$, die hier dadurch geprägt waren, dass lediglich eine einseitige Erklärung seitens der Hochschule vorlag, lässt sich in der Tat von einer Zusage ausgehen und nicht vom Angebot auf Abschluss eines öffentlich-rechtlichen Vertrages ${ }^{8}$. Sieht man, wie die Vorinstanz, in der Rückzahlungsverpflichtung eine Nebenbestimmung gem. § 36 BayVwVfG in Form einer auflösenden Bedingung, so muss in formeller Hinsicht dasselbe wie für den Verwaltungsakt oder die Zusage gelten, insbesondere ist auf die Anforderungen des $\S 37$ BayVwVfG hinzuweisen. Im Zusageschreiben des Präsidenten der Hochschule waren die für die Klägerin vorgesehenen Besoldungsbezüge (Grundbesoldung nebst Leistungsbezügen) unter I. erwähnt. Es folgten Regelungen zur Ruhegehaltsfähigkeit (II.), zu Besoldungsanpassungen (III.), Sonderzahlungen, Familienzuschlägen, Umzugskosten und Trennungsgeld (IV.). Unter V. ging das Schreiben auf Gestaltungsmöglichkeiten der WBesoldung ein. Am Ende - nahezu versteckt - findet sich der Passus auf die streitgegenständliche Rückforderung der Leistungsbezüge unter „Weitere Hinweise“, drucktechnisch war dieser Passus nicht hervorgehoben und fiel daher auch nicht auf. In zivilrechtlichen Kategorien hätte die Annahme einer überraschenden Klausel gem. $\S 305 \mathrm{c}$ BGB nahe gelegen. Auch wenn das VwVfG keine dem § 305c BGB vergleichbare Regelung kennt, könnten auf den ersten Blick Bedenken gegen die Wirksamkeit der Rückzahlungsklausel bestehen, etwa wegen eines irreführenden Verhaltens der Hochschule, indem sie die uneingeschränkte Geltung der Leistungsbezüge durch die textlich getrennte Unterbringung der Rückzahlungsklausel verschleierte und somit bei der Klägerin eine Fehlvorstellung hervorrief ${ }^{9}$. Der BayVGH hat diese Frage letztlich mit dem Hinweis offen gelassen, dass gegen die Rückzahlung von Berufungs- und Bleibe-Leistungsbezügen durch Bescheid keine Bedenken bestünden ${ }^{10}$.

In rechtlicher Hinsicht ist dem zwar zu folgen, es bleibt gleichwohl der Beigeschmack fehlender Transparenz: Wenn das Berufungsangebot schon einen Hinweis auf die Rückzahlungsverpflichtung aufnimmt, dann gehört er vom inhaltlichen Zusammenhang her an die Stelle, an der die Gewährung der Berufungs-Leistungsbezüge festgehalten wird.

\section{Rückforderung nur bei unbefristeten Leistungsbezügen}

Überzeugend nimmt der Verwaltungsgerichtshof in den Entscheidungsgründen bei Auslegung von Art. 70 Abs. 3 S. 2 BayBesG an, dass die Vorschrift nur für eine Rückforderung unbefristeter Leistungsbezüge als Ermächtigungsgrundlage herangezogen werden kann.

\footnotetext{
8 So auch VG Würzburg v. 24.11.2015, W 1 K 14.811, juris; vgl. hierzu Detmer, a.a.O., Kap. 4, Rdnr. 113; Wertheimer, OdW 2015, 147, $152 \mathrm{mwN}$.

$9 \quad$ Vgl. dazu Stelkens/Bonk/Sachs, 9. Aufl. 2018, § 36 Rn. 34.

$10 \quad$ Unter Hinweis auf BVerwG v. 12.04.2017, 2 C 16.16, juris.
} 
Zutreffend sind in diesem Zusammenhang auch die Ausführungen, dass der Gesetzgeber mit besagter Ermächtigungsnorm nicht auch einen Stellenwechsel ohne Wechsel des Dienstherrn sanktionieren wollte - zur Erinnerung: die Klägerin wechselte an eine andere Hochschule innerhalb des gleichen Bundeslandes.

An dieser Stelle hätte der Verwaltungsgerichtshof "das Buch schließen können“, die Feststellung, dass der Rückforderungsbescheid von Art. 70 Abs. 3 S. 2 BayBesG nicht gedeckt ist, wäre bereits ausreichend gewesen. Zu begrüßen ist deshalb, dass unter $1 \mathrm{~d}$ ) der Gründe weitere Ausführungen folgen. Das gilt insbesondere für die Feststellung, dass die Anwendbarkeit der Norm auf alle Fälle, in denen ein Professor oder eine Professorin innerhalb von drei Jahren seit Gewährung der Berufungs-Leistungsbezüge an eine andere Hochschule wechselt, mit höherrangigem Recht nicht vereinbar wäre, die Bestimmung vielmehr einer verfassungskonformen Auslegung bedürfe. Die dann folgenden Ausführungen würden nämlich gerade dann helfen, wenn der Gesetzgeber in Bayern die Rückforderungsbestimmung des Art. 70 Abs. 3 auf befristete Leistungsbezüge erweitern sollte. Vor diesem Hintergrund wirkt die Entscheidung gleichsam „in die Zukunft“.

\section{Verfassungskonforme Auslegung}

Wenn der Verwaltungsgerichtshof hierbei festhält, dass für eine Rückzahlungsverpflichtung von Berufungsleistungsbezügen bei einem Verlassen der Hochschule kein Raum besteht, wenn diese Leistungsbezüge für eine nach Ernennung im Dienstverhältnis erbrachte Leistung bezahlt werden ${ }^{11}$, so verdient das uneingeschränkte Zustimmung.

a) In diesem Zusammenhang hat das Gericht auch der Auffassung des VG Würzburg in der ersten Instanz eine Absage erteilt, die eine Parallele zur Rechtsprechung des Bundesverwaltungsgerichts zur Frage der Rückzahlung von Anwärterbezügen bzw. Anwärtersonderzuschlägen bei Nichteinhaltung einer bestimmten Bleibeverpflichtung von Beamtenanwärtern oder Beamten gezogen hatte. Weil das BVerwG dort Mindestdienstzeiten von 5 Jahren im Hinblick auf Art. 12 Abs. 2 GG für zumutbar erachtet hat, hielt das Verwaltungsgericht Würzburg eine dreijährige Bindungsdauer auf Grundlage der Vergabegrundsätze der Hochschule (noch) für verhältnismäßig. Der Verwaltungsgerichtshof hält dem in den Entscheidungsgründen entgegen, dass die Hochschule mit den Leistungsbezügen nicht in die Person der Klägerin „investiert“, sondern ihr durch Gewährung der höchsten Stufe der Berufungs-Leistungsbezüge ein Entgelt für zu erbringende Leistungen zugesagt habe. 
Auf die Rechtsprechung zu Ausbildungs- und Fortbildungsaufwendungen lässt sich hier zurecht nicht rekurrieren: Nach $\S 59$ Abs. 5 BBesG, dem im Übrigen Art. 75 Abs. 2 BayBesG ähnelt, kann für Anwärter, die im Rahmen ihres Vorbereitungsdienstes ein Studium ableisten, die Gewährung der Anwärterbezüge von der Erfüllung von Auflagen abhängig gemacht werden. Die ratio dieser Norm hat mit der hiesigen Fragestellung nichts gemein: Nach Sinn und Zweck soll § 59 Abs. 5 BBesG sicherstellen, dass Anwärter, die zunächst im Rahmen ihres Vorbereitungsdienstes an einer Hochschule studieren und nach dem Studienabschluss nicht mehr bereit sind, als Beamte im Dienst eines öffentlich-rechtlichen Dienstherrn zu verbleiben, keine finanziellen Vorteile gegenüber anderen Studierenden erlangen ${ }^{12}$. Maßgeblich für die Rechtfertigung einer Auflage iSd $\S 59$ Abs. 5 BBesG ist ferner, dass ein Studium im Rahmen eines Beamtenverhältnisses gefördert wird und der Beamte auf Widerruf während des Studiums insbesondere einen Anspruch auf Besoldung hat. Diese kostenaufwendige Form der Ausbildung im Rahmen des Vorbereitungsdienstes privilegiert die Anwärterstudierenden im Vergleich mit anderen Beamten auf Widerruf im Vorbereitungsdienst und im Vergleich mit Studierenden, die während ihrer Ausbildung keine Bezüge nach dem BBesG erhalten ${ }^{13}$.

Diese Interessenlage unterscheidet sich maßgeblich von der hier vorliegenden. Während der Anwärter Bezüge nach dem BBesG erhält, ohne dem Dienstherrn eine (Dienst-)Leistung zu schulden und daher mit einer Rückforderung belegt werden kann, wenn er nicht für eine gewisse (Armortisations-) Zeit nach Abschluss der Ausbildung beim Dienstherrn verbleibt, hatte die Klägerin in den 21 Monaten, in denen sie an der Hochschule Forschung und Lehre in ihrem Fach gepflegt hat, eine echte Gegenleistung erbracht. Darauf hat der Verwaltungsgerichtshof bei der Bewertung der Stufen der Vergabegrundsätze der Hochschule zu Recht abgehoben. Man kann dies noch zuspitzen: Die Leistungsbezüge der Vergabegrundsätze definieren den „Marktwert“, der der Klägerin zugemessen wurde. Auf Grundlage dieses Marktwerts hat sie mit Aufnahme ihres Dienstes Forschungs- und Lehrleistungen und damit Gegenleistungen in einem echten do-ut-des-Verhältnis erbracht. Diese Gegenleistung hat durch den Umstand, dass die Klägerin die Hochschule nach 21 Monaten wieder verlassen hat, nicht an Wert verloren.

Nichts anderes praktiziert der Verwaltungsgerichtshof, indem er die arbeitsrechtlichen Grundsätze überträgt, wonach Regelungen unzumutbar sind, die in unzulässiger Weise die freie Arbeitsplatzwahl beschränken, die auch das Recht des Beamten einschließt, den einmal gewählten Arbeitsplatz beizubehalten, aufzugeben oder zu wechseln und die den gleichen Schutz genießt wie die Freiheit der Berufswahl. Dabei entspreche es, so der

\footnotetext{
12 BVerwG v. 13.09.2011, 2 A 9/00, ZTR 2002, 95; v. 03.07.2009, 2 B 13/09, juris; vgl auch BVerwG v. 27.02.1992, 2 C 28/91, NVwZ 1993, 372; gleichermaßen für die Rückforderung von Ausbildungskosten eines ehemaligen Offiziers auf Zeit: BVerwG v. 12.4.2017, 2 C 16.16, BVerwGE 158, 364. 
Verwaltungsgerichtshof, gesicherter arbeitsrechtlicher Dogmatik, dass für Sonderzahlungen mit Entgeltcharakter Rückzahlungsvorbehalte unzulässig sind, wenn Gegenstand der Zahlung Entgelt für bereits geleistete Arbeit ist ${ }^{14}$.

b) Abgesehen davon hat das Verwaltungsgericht erstinstanzlich bei der Parallele zur Rückzahlung von Ausbildungs- und Fortbildungskosten zwei weitere Aspekte außer acht gelassen: Zum einen unterfallen Anwärterbezüge nicht dem verfassungsrechtlich verankerten Alimentationsprinzip nach Art. 33 Abs. 5 GG ${ }^{15}$, weshalb die Anforderungen an die Zumutbarkeit einer Rückforderung allein deshalb schon niedriger liegen. Zum anderen ist die Rückzahlungspflicht auf den Teil der Anwärterbezüge begrenzt, der den Betrag in $\S 2$ Abs. 2 S. 2 des Bundeskindergeldgesetzes in der jeweils geltenden Fassung überschreitet und damit der Höhe nach limitiert ${ }^{16}$. Demgegenüber legte die der Klägerin auferlegte Rückzahlungsverpflichtung kein Limit fest.

c) Ein weiterer Aspekt scheint auf, wenn man die besoldungsrechtlichen Regelungen der Beamtenanwärter näher betrachtet. Rückzahlungsverpflichtungen sieht nämlich auch $\S$ 63 Abs. 3 BBesG, parallel Art. 78 Abs. 3 BayBesG, in Bezug auf einem Anwärter gewährte Sonderzuschläge vor. Wie aus $\S 63$ Abs. 1 BBesG ersichtlich, handelt es sich hierbei um ein finanzielles Anreizinstrument, um auf einen erheblichen Mangel an qualifizierten Bewerbern zu reagieren. Selbst dort, wo der Sonderzuschlag in erster Linie an die schlechte Bewerberlage und nicht an die besondere Qualifikation eines Anwärters anknüpft, sieht $\S 63$ Abs. 3 S. 2 BBesG noch ein „Abschmelzverfahren“ vor, indem sich der Rückzahlungsbetrag für jedes nach Bestehen der Laufbahnprüfung abgeleistete Dienstjahr um jeweils ein Fünftel verringert; ebenso ist das in Art. 78 Abs. 3 S. 2 BayBesG der Fall. Wenn demgegenüber die Rückzahlungsverpflichtung nach Art. 70 Abs. 3 BayBesG eine komplette Rückzahlung festlegt, so ist dies unverhältnismäßig.

d) Einzugehen ist noch auf die vom Verwaltungsgerichtshof vorgenommene Interessenabwägung. Die Belange der Universität, Planungssicherheit zu erhalten und den Aufwand von Berufungsverfahren in einem angemessenen Rahmen zu halten, hat er dabei hinter das Interesse einer befristet beschäftigten Professorin, Karrierechancen auf eine unbefristete Professur wahrnehmen zu können, zurückgestellt. Das könnte den Eindruck erwecken, dass bei einem unbefristet beschäftigter Hochschullehrer, der einem Ruf einer anderen Universität folgt, die Rückzahlung der gewährten Berufungs-Leistungsbezüge gerechtfertigt wäre. Dem ist nicht so: Auch in diesem Fall sind ohne Einschränkung die arbeitsrechtlichen Grundsätze, wonach für Sonderzahlungen mit Entgeltcharakter das

\footnotetext{
$14 \quad$ Unter Berufung auf BAG v. 13.09.1974, 5 AZR 48/74, NJW 1975, 278 sowie ErfK/Preis, 17. Aufl. 2017, § 611 BGB Rn. 548 mwN.

15 BVerfG v. 12.04.1972, 2 BvR 704/70, BVerfGE 33,44; BVerwG v. 27.2.1992, a.a.O.

$16 \quad$ Vgl. hierzu BayVGH v. 12.12.2014, 3 ZB 13.668, NVwZ-RR 2015, 268.
} 
Entgelt für bereits geleistete Arbeit unzulässig ist ${ }^{17}$, heranzuziehen. Abgesehen davon: Die Durchführung von Berufungsverfahren gehört zum allgemeinen Aufgabenbereich einer Universität, der hierdurch entstehende Aufwand ist Teil der Gemeinkosten - diese könne nicht im Wege einer Rückzahlungsverpflichtung auf einen Hochschullehrer, der die Universität verlässt, „umgelegt“ werden.

e) Dem Verwaltungsgerichtshof ist schließlich in seiner Bewertung zuzustimmen, dass einer verfassungskonformen Rechtsanwendung im anschließenden nicht mehr über $\S 15$ Abs. 2 BayBesG Rechnung getragen werden kann. Zur näheren Erläuterung: Die Feststellung der Rückzahlung erfolgte per Bescheid seitens der Hochschule, der konkrete Rückforderungsbetrag wurde der Klägerin über das Landesamt für Finanzen mitgeteilt. Gegen die vom Verwaltungsgericht Würzburg in erster Instanz vertretene Auffassung spricht bereits, dass das Landesamt für Finanzen ausschließlich als „Vollzugsstelle“ fungiert hat, ohne eine eigene Entscheidung getroffen zu haben. Abgesehen davon, verfügt das Landesamt für Finanzen gar nicht über die Kompetenz, im Bereich der Rückforderung von Leistungsbezügen eine eigene Sachentscheidung zu treffen. Darauf ist $\S 15$ Abs. 2 BayBesG auch gar nicht ausgelegt. Wie die Parallelvorschrift in $\S 12$ Abs. 2 BBesG handelt es sich um eine bloße Rechtsfolgenverweisung ${ }^{18}$. Im Rahmen von Billigkeitserwägungen kann dabei lediglich auf das konkrete Rückforderungsbegehren und vor allem auf die Modalitäten der Rückabwicklung und ihre Auswirkungen auf die Lebensumstände des Bereicherten abgestellt werden, wobei die Lage des Schuldners im Zeitpunkt der Rückabwicklung entscheidend ist ${ }^{19}$. Kriterien sind etwa, ob sich dieser in einer unverschuldeten Notlage befindet oder der Lebensunterhalt des Empfängers und seiner Familie auch bei Zugrundelegung einer zumutbaren sparsamen Lebensführung nicht mehr gedeckt ist. Hingegen kann das Landesamt für Finanzen im nachgelagerten Verwaltungsverfahren keine Überlegungen mehr anstellen, ob z.B. wegen der bereits zurückgelegten Dienstzeit der Klägerin nur eine Teilrückzahlung der Leistungsbezüge angemessen wäre.

\section{Unbedenkliche Anwendungsfälle von Art. 70 Abs. 3 S. 2 BayBesG ?}

Quasi im Wege eines obiter dictum führt der Senat des BayVGH zum Ende der Entscheidung hin aus, dass er "rechtlich unbedenkliche Anwendungsfälle der Ermächtigungsgrundlage des Art. 70 Abs. 3 S. 2 BayBesG für möglich halte. So könne etwa die Rückforderung von unbefristeten Bleibe-Leistungsbezügen in Betracht kommen, weil diese nicht ohne weiteres

\footnotetext{
17 Siehe hierzu die in Fn. 14 zitierte Rechtsprechung.

18 Kathke in: Schwegmann/Summer, Besoldungsrecht des Bundes und der Länder, Art. 15 BayBesG Rn. 47; BVerwG v. 28.02.2003, 2 C 2.01, BVerwGE 116; 74; Mayer in: Kommentar zum BBesG, 118. AL 2005, § 12 BBesG Rn. 27. 
als Gegenleistung für geleistete Dienste anzusehen sein werden, sondern auch allein deshalb erbracht werden können, um den (möglicherweise) abwanderungswilligen Hochschullehrer davon abzuhalten, dem Ruf einer anderen Universität zu folgen.

Versucht man, aus dieser im Urteil eher allgemein gehaltenen Aussage rechtliche Schlussfolgerungen zu ziehen, so gilt es zu differenzieren:

a) Werden seitens der Hochschule unbefristete Bleibe-Leistungsbezüge gewährt, die als Gegenleistung für zu leistende Dienste anzusehen sind, muss es bei einer verfassungskonformen Auslegung von Art. 70 Abs. 3 S. 2 BayBesG bleiben. Hier ist kein Unterschied zu befristeten Leistungsbezügen zu erkennen. Werden diese Leistungsbezüge für im Dienstverhältnis erbrachte Leistungen bezahlt, scheidet eine Rückzahlung ebenfalls aus. So sehen etwa die Vergabegrundsätze der beklagten Hochschule vor, nach dem gleichen leistungsorientierten Stufensystem, das für Berufungs-Leistungsbezüge maßgeblich ist, auch Bleibe-Leistungsbezüge zu gewähren. Diese Vergabegrundsätze sehen ferner vor, dass auch Bleibe-Leistungsbezüge in der Regel als laufende monatliche Zahlung gewährt werden. Ist das der Fall, kommt eine Rückzahlung bei einem Hochschulwechsel nicht in Betracht.

b) Ist bei den gewährten Bleibe-Leistungsbezügen kein Leistungsbezug gegeben, was im Einzelfall zunächst festgestellt werden muss, greift der vom Verwaltungsgerichtshof herangezogene arbeitsrechtliche Grundsatz nicht. Im Umkehrschluss anzunehmen, dass eine vollständige Rückzahlung in einem solchen Fall zulässig ist, begegnet jedoch Bedenken. Auch hier bedarf es im Hinblick auf Art. 12 Abs. 1 GG einer verfassungskonformen Auslegung.

Dafür kann wiederum auf die Rechtsprechung des Bundesarbeitsgerichts zurückgegriffen werden, die sich zum Bereich der Fortbildungskosten entwickelt hat. Gerade dort gibt es ähnlich gelagerte Bindungsverpflichtungen, wie sie Art. 70 Abs. 3 S. 2 BayBesG mit der 3Jahres-Frist einem Professor bzw. einer Professorin auferlegt. Der Arbeitgeber investiert mit einer Fortbildungsmaßnahme in den Arbeitnehmer, der für den Fall, dass er vor Ablauf einer bestimmten Frist das Unternehmen wieder verlässt, vom Arbeitgeber aufgewandte Kosten auf Grundlage einer Rückzahlungsklausel zurückerstatten muss. Von einem ähnlichen „Invest" wäre bei einer Hochschule auszugehen, die einem von einer anderen Hochschule umworbenen Professor eine - nicht leistungsbezogene - Bleibezulage gewährt. Wie im Arbeitsrecht ist dann zu prüfen, ob der in Art. 70 Abs. 3 S. 2 BayBesG vorgesehene Rückzahlungsvorbehalt den Professor oder die Professorin in ihrem Grundrecht auf freie Wahl des Arbeitsplatzes beeinträchtigt. In den Worten des BAG „kommt es darauf an, ob den möglichen Nachteilen für den Arbeitnehmer ein angemessener Ausgleich gegenübersteht. Dabei sind alle Umstände des Einzelfalls zu berücksichtigen. Die Rückzahlungspflicht muss vom Standpunkt eines verständigen Betrachters aus einem begründeten und zu billigenden 
Interesse des Arbeitgebers entsprechen; der Arbeitnehmer muss mit der Ausbildungsmaßnahme eine angemessene Gegenleistung für die Rückzahlungsverpflichtung erhalten haben. Insgesamt muss die Erstattungspflicht dem Arbeitnehmer nach Treu und Glauben zumutbar sein. Dabei kommt es u.a. auf die Dauer der Bindung, den Umfang der Fortbildungsmaßnahme, die Höhe des Rückzahlungsbetrages und dessen Abwicklung an"20.

Diese für den Arbeitnehmer aus Art. 12 GG abgeleiteten Überlegungen lassen sich auf beamtete Professoren oder Professorinnen übertragen. Im jeweiligen Fall ist daher zu prüfen, ob die Höhe der gewährten Bleibezulagen eine dreijährige Bindungsdauer rechtfertigen ${ }^{21}$, im Rahmen der Interessenabwägung muss, wie im Arbeitsrecht auch, die Abwicklung der Rückzahlungsverpflichtung mit zunehmendem Verbleib des Professors an der Hochschule eine Rolle spielen und kann somit zu einem angemessenen Interessenausgleich führen. Praktisch ließe sich das durch ein Abschmelzen des Rückzahlungsbetrages erreichen, der sich mit jedem Monat, den der Hochschullehrer an der Universität verbleibt, um 1/36 verringert, geht man von einer zulässigen Bindungsdauer von drei Jahren aus. Der Senat hat diesen Aspekt - wenn auch in anderem Zusammenhang - im vorliegenden Urteil gestreift. Ob ein Rückforderungsbetrag gegebenenfalls zeitanteilig abzuschmelzen wäre, so der VGH, mag sich dem Normgeber oder der Verwaltung bei der Niederlegung ihrer Verwaltungspraxis im Wege der Selbstbindung stellen.

c) Was gilt schließlich für unbefristet gewährte Berufungs-Leistungsbezüge ? Von der Ermächtigungsgrundlage des Art. 70 Abs. 3 S. 2 BayBesG werden sie erfasst. Im Ergebnis führt der Umstand, dass solche Leistungsbezüge unbefristet gewährt wurden, aber zu keiner anderen Bewertung als in den Fällen, in denen sie lediglich für einen gewissen Zeitraum zugesagt worden sind. Im einen wie im anderen Fall stellen die Leistungsbezüge Entgelt für bereits geleistete Dienste dar, das bei einem Hochschulwechsel nicht zurückgefordert werden kann.

20 BAG v. 23.04.1986, 5 AZR 159/85, DB 1986, 2135; v. 05.12.2002, 6 AZR 539/01, NZA 2003, 559; AR/Spelge, 8. Aufl. 2016, Art. 12 GG Rn. 21.

21 Vgl. ErfK/Preis, 18. Aufl. 2018, § 611 BGB Rn. 441 mwN zur Situation bei Rückzahlungsklauseln im Arbeitsrecht. 


\section{Fazit}

Die Schlussfolgerungen aus dem Urteil des Bayerischen Verwaltungsgerichtshofs zeigen, dass Berufungs- oder Bleibe-Leistungsbezüge von einem Hochschullehrer im Falle eines Hochschulwechsels nur in einem eng begrenzten Umfang zurückverlangt werden können. Das würde auch dann gelten, wenn der bayerische Gesetzgeber die Vorschrift des Art. 70 Abs. 3 S. 2 BayBesG auf befristet gewährte Leistungsbezüge erweitert. Es bleibt zu hoffen, dass diese Erkenntnis andere Bundesländer davon abhält, entsprechende Bestimmungen in ihre Besoldungsgesetze aufzunehmen und das auch hochschulpolitisch fragwürdige Instrument in Bayern bald nur eine Episode sein wird.

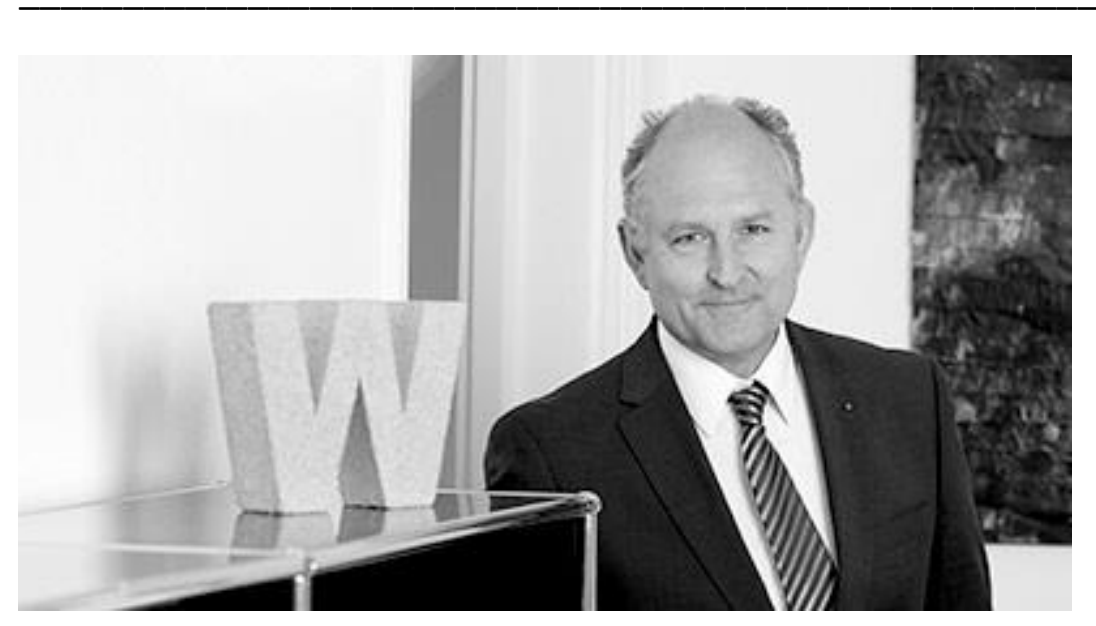

Der Autor ist Partner der Kanzlei KRAUSS LAW in Lahr/Schwarzwald und hat die Klägerin im Verfahren vor dem Bayerischen Verwaltungsgerichtshof vertreten. Zuvor war er 17 Jahre im Universitätsbereich, davon über 10 Jahre in der Hochschulmedizin tätig. Zu seinen Beratungsfeldern gehört im Bereich des Arbeitsrechts auch das Hochschulrecht. Er ist Gastmitglied der Forschungsstelle für Hochschulrecht und Hochschularbeitsrecht an der Rechtswissenschaftlichen Fakultät der Universität Freiburg. 